livraisons

d'Histoire

de l'Architecture

\section{Livraisons de l'histoire de l'architecture}

19 | 2010

Du remploi à l'éphémère

\title{
Sebald, lecteur de gares
}

Sebald - a reader of railway stations

Sebald, ein Schriftsteller, der den Raum der Bahnhöfe liest

Julia NYIKOS

\section{(2) OpenEdition}

\section{Journals}

Édition électronique

URL : http://journals.openedition.org/lha/238

DOI : 10.4000/lha.238

ISSN : 1960-5994

Éditeur

Association Livraisons d'histoire de l'architecture - LHA

Édition imprimée

Date de publication : 10 juin 2010

Pagination : 61-76

ISSN : $1627-4970$

Référence électronique

Julia NYIKOS, "Sebald, lecteur de gares », Livraisons de l'histoire de l'architecture [En ligne], 19 | 2010, mis en ligne le 10 juin 2012, consulté le 21 avril 2019. URL : http://journals.openedition.org/lha/238 DOI : $10.4000 /$ /ha. 238

Ce document a été généré automatiquement le 21 avril 2019

Tous droits réservés à l'Association LHA 


\title{
Sebald, lecteur de gares
}

\author{
Sebald - a reader of railway stations \\ Sebald, ein Schriftsteller, der den Raum der Bahnhöfe liest
}

Julia NYIKOS

1 Parmi les textes littéraires contemporains, peu consacrent autant d'attention à l'architecture, à la description littéraire d'espaces architecturaux et à l'image photographique de constructions urbaines que le dernier roman de W. G. Sebald ${ }^{1}$, intitulé Austerlitz $^{2}$. De nombreuses critiques ont développé une lecture métaphorique des éléments architecturaux décrits dans le roman, tout en proposant des rapprochements analogiques entre le parcours du protagoniste et les valeurs symboliques des édifices présentés par le texte.

2 L'objectif du présent article consiste à examiner la représentation littéraire des espaces et des édifices, et à voir comment le sens multiple de l'architecture influence l'organisation structurelle du roman. On se concentrera sur la représentation des bâtiments de gare qui apparaissent de manière récurrente dans Austerlitz.

3 Le livre de Sebald se compose d'une succession de dialogues entre le narrateur et Austerlitz, le personnage éponyme du roman. Ce dernier se passionne pour l'architecture et étudie, tout au long du texte, des édifices de gare en Belgique, en Angleterre, à Paris ou à Prague. En expliquant les spécificités des stations et terminus ferroviaires, il intègre à ses propos des observations concernant d'autres lieux servant de contexte aux conversations: palais de justice, archives nationales, hôtels, bibliothèques et fortifications. Or, ces édifices, qui guident la narration, se révèlent les points clés d'un parcours que le personnage principal effectue pour découvrir sa propre histoire et son identité ignorée ou perdue (ill. 1). 
III. 1 : Espace de dialogues

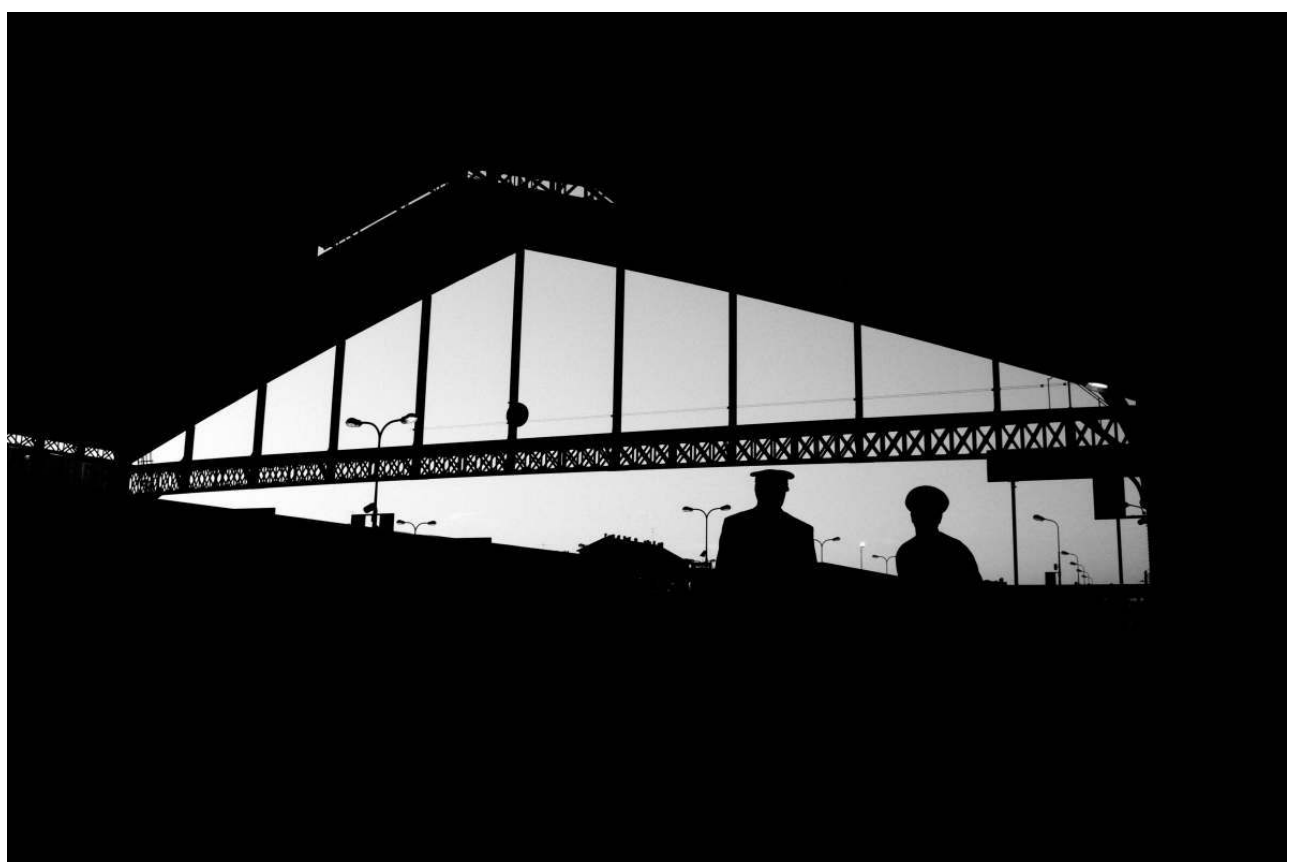

Cl. Imre Miszlay

Les gares auxquelles Austerlitz s'intéresse le plus datent du XIX ${ }^{\mathrm{e}}$ siècle : gare centrale d'Anvers, Liverpool Street Station, Broadstreet Station, gare du Nord et gare de l'Est à Paris. Les analyses s'attachent régulièrement à la salle des pas perdus, aux salles d'attente et aux espaces centraux pourvus de coupoles, de verrières et de miroirs. Ainsi, on examinera certains mécanismes de fonctionnement des gares et de leurs espaces intérieurs, afin de voir comment le texte « lit » ces constructions et à quel point la logique architecturale parvient à déterminer celle de la narration et de la composition du roman. Il faut également examiner certaines caractéristiques de ces gares datant de la deuxième moitié du XIX ${ }^{\mathrm{e}}$ siècle, tout en cherchant à comprendre comment le texte littéraire d' Austerlitz les met en scène, les exploite ou les amplifie au long du récit.

\section{L'entrée des chemins de fer}

5 Comme la souffrance physique constitue un leitmotiv du texte, notamment en rapport avec l'expérience d'espaces urbains et architecturaux, il convient d'évoquer brièvement les relations que le corps humain entretient avec les voies ferrées dès l'apparition de celles-ci.

6 L'entrée des chemins de fer à l'intérieur des villes implique un impact direct sur les habitants mêmes. Au début du XIX ${ }^{\mathrm{e}}$ siècle, elle conduit à la détérioration considérable et rapide de la santé des populations plus pauvres (notamment ouvrières) et des conditions hygiéniques de leurs logements surpeuplés, rudimentaires et défectueux situés dans la proximité des zones ferroviaires. Autrement dit, les corps amoncelés, entassés et enfermés, corps malsains, maladifs et contaminés, se révèlent être en rapport direct avec les voies ferrées qui dévorent l'air et l'espace habitable des villes ${ }^{3}$. L'installation urbaine des chemins de fer s'effectue dans un climat de conflit avec les habitants; à part les 
changements sociaux, esthétiques ou politiques, les corps humains se trouvent affectés avant tout physiquement.

7 Outre la détérioration des conditions d'habitation de presque toutes les couches sociales, l'entrée des chemins de fer dans les villes réorganise le paysage citadin dès le moment où les usines et les voies ferrées deviennent les nouveaux noyaux des espaces habités. Au lieu de suivre des plans d'aménagement conformes à des conceptions plus traditionnelles de structures urbaines, les chemins de fer déterminent les formes et les limites des agglomérations, introduisant coupures et fragmentations à l'intérieur de quartiers et de territoires autrefois organiquement reliés. Séparation, morcellement et disjonction accompagnent donc les aménagements industriels des usines et des voies ferrées dans le tissu urbain.

Le conflit prend également une ampleur historique dans la mesure où les chemins de fer entrant au cœur des cités, les postes de triage, les hangars, les dépôts et les gares deviennent des constantes du paysage des centres-villes (ill. 2) qui conservaient et reflétaient jusqu'alors l'unité d'une ville remontant parfois aux temps médiévaux. La mémoire des cités d'antan se trouve souvent brouillée sinon détruite par les aménagements industriels qui constituent désormais l'unique centre d'intérêt au détriment des centres historiques.

III. 2 : Paysage du centre-ville

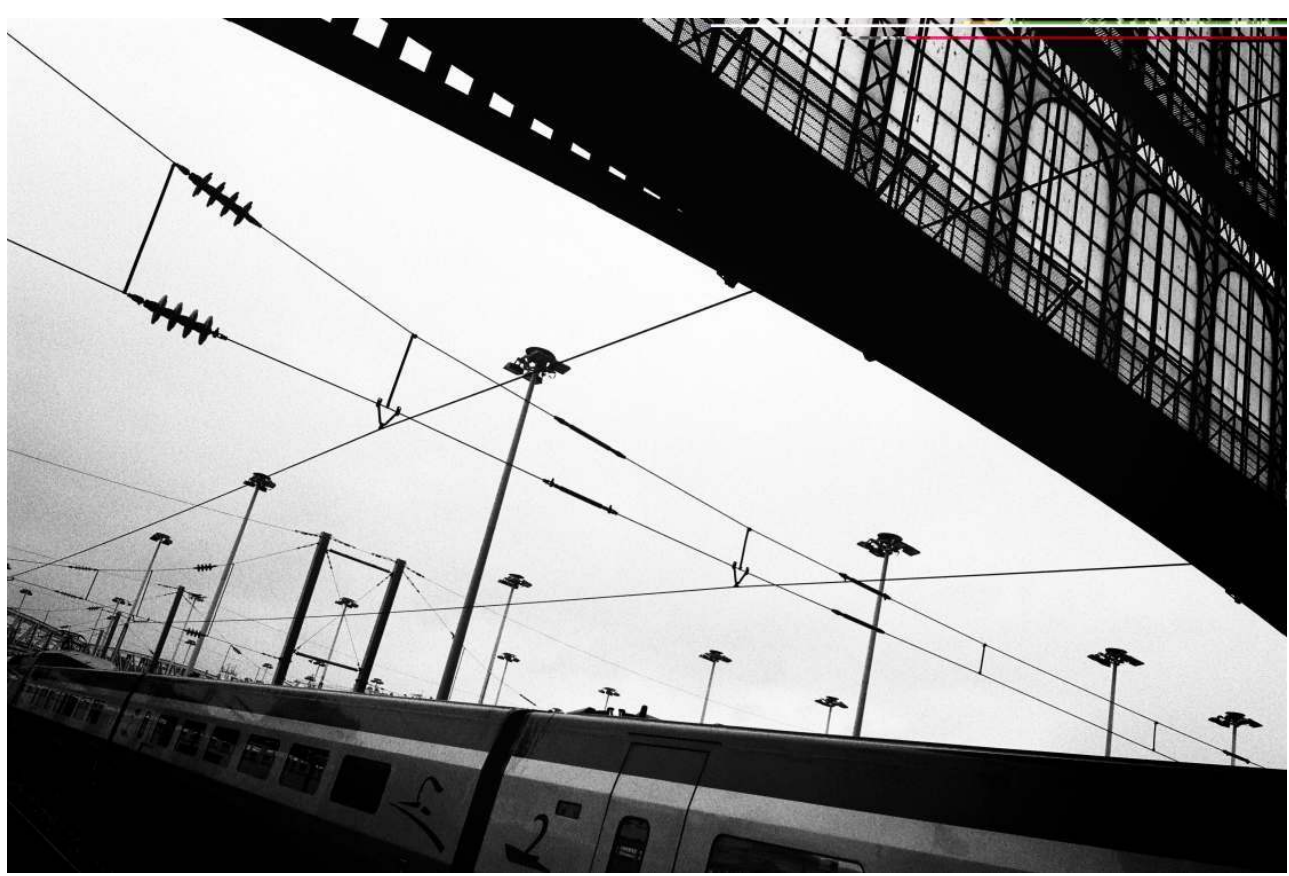

Cl. Imre Miszlay

\section{La gare des souffrances}

Dans le roman, la problématique des mémoires et de l'héritage des lieux est exprimée de façon parabolique avec la superposition de deux tableaux dressés respectivement à partir de Liverpool Street Station et de Broad Street Station. 
10 D'après le récit, à l'emplacement de la première gare se trouvaient autrefois des terrains marécageux et à la place du bâtiment central de la gare, un prieuré de l'ordre de SainteMarie de Bethléem s'élevait jusqu'au XVII ${ }^{e}$ siècle, duquel dépendait un « hospice d'aliénés et autres nécessiteux ». Austerlitz explique qu'il lui arrive d'imaginer « où avaient pu se trouver les cellules des pensionnaires de cet asile » et que, souvent, il s'était « demandé si la peine et les souffrances qui s'y étaient accumulées au fil des siècles avaient jamais cessé d'être, si aujourd'hui encore, [...], il ne nous arrivait point de les traverser en allant et venant dans la salle des pas perdus ou en montant et descendant les volées d'escaliers ${ }^{4} \ldots$ ” Le récit lie au bâtiment de la gare la souffrance ; l'emplacement de la gare semble porter en soi des réminiscences négatives et, plus particulièrement, l'atmosphère des asiles d'autrefois.

11 En enchaînant cette histoire à celle de Broad Street Station, le récit propose un nouveau rapprochement entre les citadins et la gare. Il explique que, lors des fouilles pour la construction de la gare, les archéologues avaient trouvé des centaines de cadavres à son futur emplacement et qu'un quartier de population misérable s'était ensuite installé à l'endroit de cet ancien cimetière. Le récit configure donc un lieu, un emplacement hanté par la mort et la misère humaine, où il semble impossible de détacher de l'image des locaux de gare non pas tant les vestiges d'édifices précédents, mais des souffrances physiques préfigurant celles qu'ont suscitées les premières phases d'industrialisation.

\section{La violence de l'architecture}

12 Le rapport des chemins de fer et de l'architecture des gares aux corps humains peut être interprété autrement que par le seul biais historique. Le conflit comme matrice entre les voyageurs et les manifestations architecturales du développement industriel doit être révélateur d'un rapport d'interdépendance qui permet de réinterpréter leur relation tendue. Les souffrances infligées aux populations résidant à proximité des zones ferroviaires n'ont montré que des nuisances et brutalités physiques. Or, l'intensité des relations peut aussi indiquer des dialogues plus complexes et plus subtils entre les éléments qui s'affrontent dans ce conflit.

13 Examinant les rapports entre corps humains et corps d'édifices, l'architecte Bernard Tschumi propose de les interpréter en termes de violence. La violence se révèle pour lui inhérente à la définition même de l'architecture dans la mesure où celle-ci désigne ce rapport de réciprocité entre espace et action dans lequel l'un contraint l'autre à certaines déterminations. Dans cette lecture, la violence devient un facteur créateur qui indique dans quelle mesure un édifice et un corps humain peuvent interagir et devenir inséparables. Ainsi, les corps humains, en entrant dans un bâtiment, infligent une violence à l'équilibre de l'ordre précis, pur et géométrique des espaces. De façon inverse, la torture que l'architecture peut exercer sur le corps humain va des couloirs étroits accueillant des foules immenses, des chambres trop petites ou trop grandes, des plafonds trop bas ou trop hauts à des incommodités spatiales plus complexes, comme des escaliers dangereux, des espaces informes psychologiquement éprouvants ou ceux entièrement dépourvus d'échos 5 .

Le texte romanesque met également en valeur une sorte de violence créatrice entre éléments architecturaux et corps humains. En présentant la Liverpool Street Station, un passage décrit " une foule innombrable s'échappant des trains ou s'y engouffrant», qui 
"s'écoulait en flux qui se rejoignaient, s'écartaient, s'amassaient aux barrières et aux étranglements comme l'eau au pied d'une retenue ${ }^{6} »$. L'architecture intérieure s'adapte mal aux usagers, et les voyageurs se voient obligés de s'accommoder des formes de l'espace de la gare. La foule est en quelque sorte inerte, se heurte aux obstacles physiques de l'enceinte. Elle forme un flux homogène où les individus et leur parcours personnel s'effacent. En étant exposés aux contraintes de l'espace, les gens perdent dans la gare toute individualité ou volonté personnelle. Par ailleurs, cette technique narrative qui consiste à remplacer l'approche anthropocentrique de l'espace par une description où le flux de la foule épouse les formes d'un espace intérieur n'est pas une invention de Sebald

Notre-Dame de Paris

\section{Corps de gare et corps humains}

Le texte romanesque met en scène les transformations effectuées par les aménagements ferroviaires au travers d'une approche double. Il aborde l'histoire de ce qui était un ancien champ de blanchissage et un cimetière. L'actuel terrain de Broad Street Station abritait en effet auparavant non seulement des champs pour étaler le linge à blanchir, mais aussi les morts à inhumer. Le vaste cimetière où les corps se trouvaient de plus en plus entassés les uns sur les autres devait donc accueillir, vers la fin du XIXe siècle, une station ferroviaire. Lors des fouilles visant, un siècle plus tard, à aménager une station de taxis, les os et les débris se sont de nouveau fait jour. Il apparaît ainsi que « dans chaque mètre cube de débris retirés de la fosse on a trouvé en moyenne les ossements de huit individus. Par-dessus cette couche de terre ainsi parsemée de la poussière et des os de cadavres décomposés, la ville, aux $\mathrm{XVII}^{\mathrm{e}}$ et $\mathrm{XVIII}{ }^{\mathrm{e}}$ siècles, s'était développée en un enchevêtrement de plus en plus dédaléen de ruelles et de maisons malsaines ${ }^{7}$.»

De cette description se dégage l'image d'une ville bâtie sur un cimetière et sur un énorme amoncellement de corps humains. Une photo insérée dans le texte représente des squelettes dans un fossé boueux. L'autre image, en revanche, donne un dessin technique représentant, sous forme de plan, un faisceau de rails à Liverpool Station.

À partir de ces deux représentations de la gare, des gares anglaises, se profilent d'une part l'histoire configurée dans ces lieux de souffrances et de corps morts et, d'autre part, un organisme industriel exactement ordonné qui, malgré son caractère inhumain, évoque l'anatomie harmonieuse du corps de l'homme. Tentons d'interpréter ces antagonismes.

19 Il semble que le texte cherche à bouleverser la séparation entre corps humain et corps de gare. La description selon laquelle la foule des voyageurs emprunte la forme de l'espace intérieur du hall d'arrivée de la gare montre que le corps humain se conforme à l'espace et s'identifie en quelque sorte avec l'édifice. Les rails espacés à cause des quais puis leur faisceau de plus en plus serré en sortant de la gare imitent à leur tour, comme le souligne le texte aussi, les muscles et les tendons humains. Dans un mécanisme inverse, c'est le corps inerte qui prend l'apparence du corps animé. Autrement dit, un rapport d'intimité charnelle semble lier les lieux de la gare aux corps des gens qui entrent en contact avec l'emplacement ou le bâtiment de celle-ci. Le sujet usager et l'objet dont il est fait usage ne peuvent pas être entièrement distingués: une relation d'interchangeabilité empêche d'effectuer des identifications bien nettes. Le sujet et l'objet, l'utilisateur et l'utilisé se confondent, de même qu'il devient problématique de délimiter le corps souffrant même. 
Les corps de l'asile d'antan, les corps inhumés à l'emplacement de la gare et le corps même de la gare se superposent. Leur relation est complexe, il paraît impossible d'établir un seul type de rapport entre ces éléments. Les « faisceaux de muscles » des rails ne peuvent pas être une simple extrapolation ou une expression métonymique des corps humains dont la souffrance est rattachée à la gare ou à son emplacement, notamment parce que ces «faisceaux de muscles» forment une image anatomique ordonnée, harmonieuse, équilibrée qui traduit la connotation d'un corps en bonne santé.

Les chemins de fer compris comme des corps, des ensembles merveilleusement ordonnés (ill. 3), permettent donc de mettre en lumière la question de la qualité de la relation entre construction bâtie et corps humains. Comme les exemples cités ci-dessus le démontrent, les interactions ne peuvent pas être réduites à un rapport sujet-objet. Cette complexité doit maintenant être interprétée à la lumière du choix et du fonctionnement des formes architecturales ferroviaires dans leur contact avec les usagers des gares.

\section{3 : Ensembles merveilleusement ordonnés}

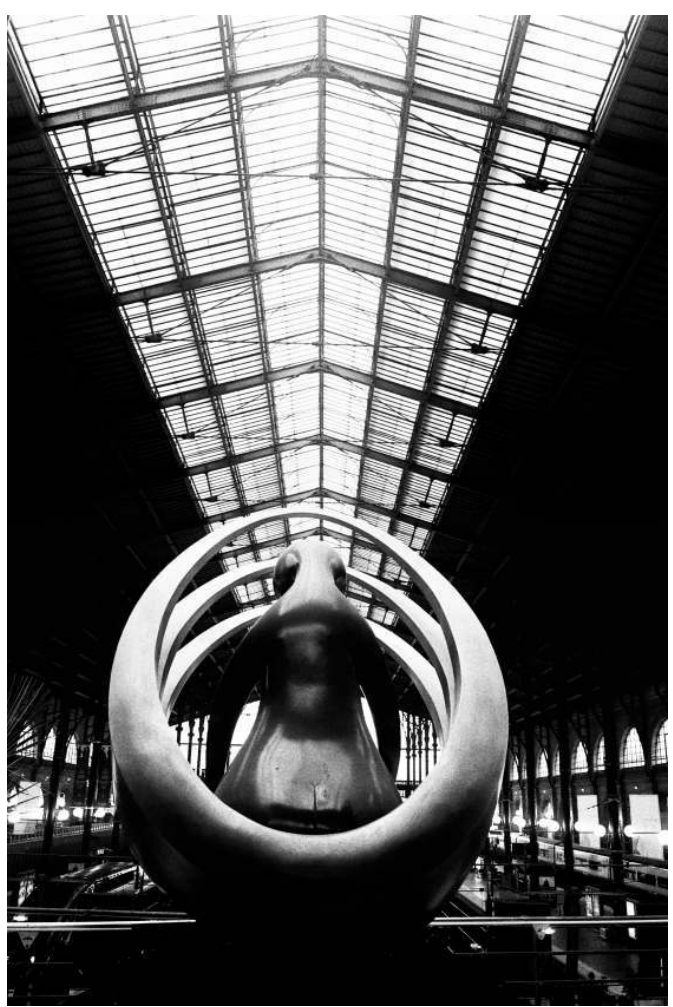

Cl. Imre Miszlay

\section{L'héritage des rotondes}

21 Les réactions correspondant à l'époque de la construction des premières gares traduisent souvent, dans la littérature comme dans les témoignages historiques, une certaine hostilité et frayeur à l'égard des chemins de fer bruyants, écrasants, puissants qui servent désormais de contrepoint au mode de vie calme et lent des contrées rurales. Afin de faire oublier les bouleversements - dans la structure des villes comme dans celle des habitudes de vie - la « quasi-totalité des bâtiments de gare du XIX ${ }^{\mathrm{e}}$ siècle prendra les apparences de temples grecs et de thermes romains, de basiliques romanes et de cathédrales gothiques, 
de châteaux Renaissance et d'abbayes baroques $»^{8}$ ou encore de palais dans le style de la Renaissance florentine.

Suivant ces principes, les gares du XIX ${ }^{e}$ siècle - et en cela celles qu'évoque le roman Austerlitz ne font pas exception - abritent souvent des éléments épousant une forme circulaire. Les coupoles et les rotondes des salles d'attente ou des salles des pas perdus répondent à cette articulation géométrique, faisant référence à un ensemble de constructions circulaires marquantes de l'histoire de l'architecture, commençant par les tholos antiques, et passant par les tombeaux étrusques, le Panthéon romain, les mausolées funéraires, les thermes romains, les temples de Vesta, ainsi qu'un grand nombre d'édifices médiévaux et renaissance, tels, par exemple, les rotondes mariales ou les basiliques chrétiennes.

Ces citations architecturales impliquent des conséquences notables sur les valeurs symboliques et sur l'usage de ces bâtiments de gare. En mobilisant les références à l'architecture funéraire et religieuse, ces constructions dotent les espaces intérieurs de significations contradictoires. Au lieu d'exprimer l'idée du mouvement, de la vitesse, du voyage, de la découverte, notions habituellement associées aux chemins de fer depuis le début de leur apparition, ces rotondes invitent à la contemplation, au recueillement et à la méditation, sans pour autant proposer de contenu approprié à ce type d'activité ou d'attitude. L'apparence du sacré se réfère sans doute plutôt à la nouvelle religion de l'époque, en l'occurrence à la réunion du commerce, de l'industrie et de la colonisation. Or, même pour les voyageurs du XIX ${ }^{\mathrm{e}}$ siècle, ce déplacement de sens des édifices religieux ne pouvait être perçu que de manière indirecte et métaphorique, tant la configuration intérieure montre de discordance par rapport à la fonction du lieu. L'architecture des rotondes de gare se révèle trompeuse, elle prépare les récepteurs à quelque chose dont elle est incapable d'assurer les conditions. La récollection, à laquelle l'univers clos d'une forme circulaire surmontée d'une coupole peut inviter, s'adapte difficilement au désordre et au chaos régnant au niveau du sol, au va-et-vient et au bruit constants des voyageurs.

Le choix formel des rotondes comprend d'autres dissemblances conceptuelles encore. Le cercle, par son absence d'orientation, permet d'articuler des directions différentes. La rotonde, représentant une certaine perfection et simplicité géométrique, «définit la clôture pour mieux accueillir du sens $»^{9}$. Or, l'apparente liberté de cette forme impose des contraintes importantes aux architectes, tout comme aux voyageurs. Car en affichant le principe d'absence d'orientation, les rotondes des gares contredisent le fondement même du système ferroviaire, à savoir le déplacement d'un point défini vers un objectif déterminé. Au lieu de trier les gens selon les destinations et le sens du mouvement (départ ou arrivée), ces halls circulaires confrontent et condensent la multitude des déplacements et des directions.

Pour mieux comprendre les mécanismes internes de telles constructions circulaires, on renvoie à un des exemples type de cette forme architecturale. La Villa Rotonda de Palladio avec sa symétrie axiale et la répétition de la disposition de chaque côté conduit à une monotonie et à une infinité qui créent un malaise croissant chez le promeneur. Elle transforme l'espace circulaire en un instrument presque sadique qui déstabilise celui qui s'y déplace ${ }^{10}$. Derrière l'harmonie cristallisée de la haute Renaissance, une architecture "violente » se profile dans la mesure où, au lieu de mettre en place une disposition permettant un usage multiple du lieu, elle attire l'attention sur ses propres qualités en les exhibant constamment. Corps humain et corps architectural entrent si intensément en dialogue ici, que toute autre action s'y trouve assujettie. 
À un degré moindre, le même effet se reproduit dans tout espace circulaire et symétrique; en gare, les coupoles et les rotondes des salles d'attente monopolisent ainsi l'attention du corps des voyageurs, elles captivent en quelque sorte ceux qui n'y cherchent qu'une issue. L'absence d'orientation, découlant du choix formel des salles d'attente rondes, peut conduire à un processus de désorientation si l'aménagement intérieur reste monotone.

\section{Absence d'orientation}

La première rencontre entre le narrateur et le protagoniste du roman s'effectue précisément dans la salle des pas perdus sous le grand hall à coupole. Austerlitz est planté dans la salle d'attente en train d'examiner «l'alignement des fenêtres, les pilastres cannelés ou autres détails et parties de l'édifice $»^{11}$ (ill. 4).

\section{4 : « Alignement des fenêtres et ...autres détails »}

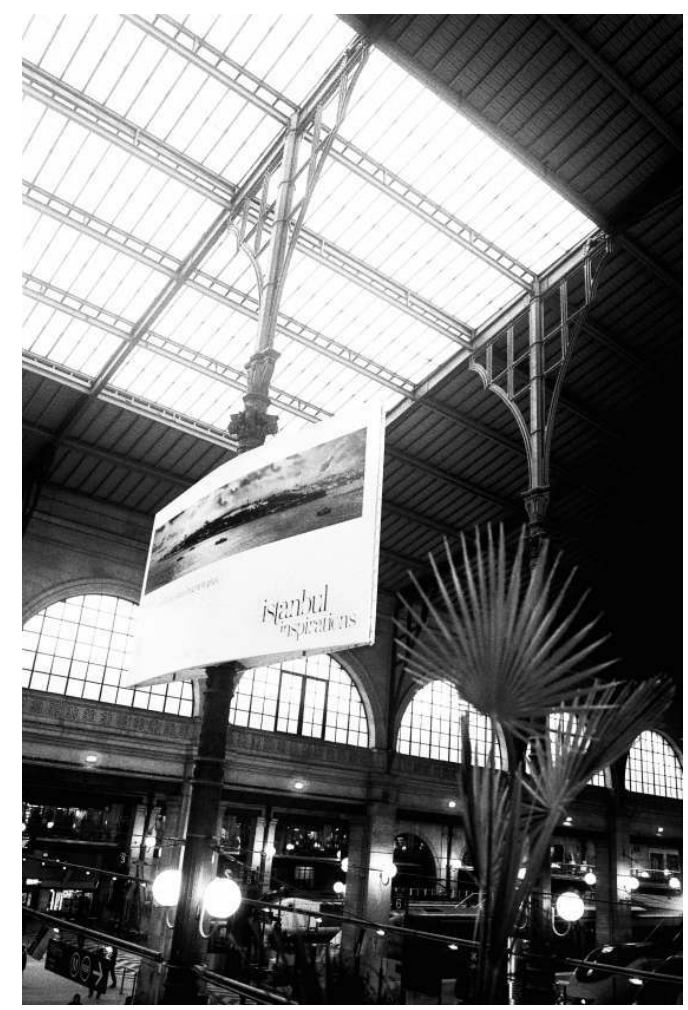

Cl. Imre Miszlay

En opposition aux autres voyageurs, pour qui ce lieu sert l'attente ou le passage, le personnage principal du roman parvient, par son immobilité, à se soustraire aux lois inhérentes à cette construction, instrument de désorientation. Austerlitz choisit donc de se fixer un point dans la rotonde, échappant ainsi aux difficultés de l'orientation. C'est en déconsidérant la fonction première de la salle des pas perdus que notre protagoniste parvient à arrêter le tourbillon et l'éternel retour de la monotonie et du non-sens du lieu. C'est aussi en levant son regard vers la coupole qu'il se détache de la sphère du désordre pour pouvoir contempler, apprécier l'ordre. 

était omniprésent, un changement considérable s'opère dans le dernier livre de l'auteur. En effet, pour le personnage d'Austerlitz, c'est l'impossibilité de trouver son chemin qui devient de plus en plus pénible. Cet état de perte permanente semble être dangereux et peut conduire à la folie. Par rapport aux autres personnages sebaldiens, c'est donc la désorientation qui détermine Austerlitz. Or, dans ce texte, le fait d'être perdu va bien audelà de l'absence ou de la perte des repères topographiques. En effet, ne pas savoir où l'on se trouve signifie, pour Austerlitz, ne pas savoir qui il est ${ }^{13}$. En choisissant comme leitmotiv une forme architecturale fonctionnant par définition comme un instrument de désorientation et de travestissement (on reviendra sur ce point), le roman projette, à travers une interprétation subtile des bâtiments de gare, une image synthétique des problèmes architecturaux dans le parcours - narratif et ainsi temporel - du protagoniste du récit. qui associent souvent les rotondes aux monuments funéraires, ainsi que le départ vers la mort, la désorientation s'accomplit également dans le roman de Sebald avec des connotations funèbres. En parlant des mites qui peuplent en été la maison du protagoniste, celui-ci exprime qu'«il n'est pas rare que l'un ou l'autre de ces insectes nocturnes s'échappe du petit bout de jardin derrière [sa] maison pour venir s'égarer à l'intérieur. Quand je me lève le matin, je le vois alors posé, immobile, quelque part sur un mur. Ils savent, je crois, dit Austerlitz, qu'ils se sont perdus, car [...] ils restent là prostrés jusqu'à leur dernier souffle. [...] Il m'arrive de me demander quelle sorte de peur et de douleur elles peuvent bien ressentir dès lors qu'elles se sont égarées ${ }^{14} »$. Dans le roman comme dans les gares, c'est à l'intérieur, dans un espace clos que l'on peut s'égarer et mourir. Le poids de l'architecture, ses références iconographiques ainsi que ses mécanismes internes, loin d'être anodins, préfigurent en quelque sorte, à travers la figure de l'égarement, la mort.

Dans la lecture de Sebald, les gares et les expériences que le voyageur ou le spectateur peut y faire, se composent d'éléments multiples, parmi lesquels les effets de lumière jouent un rôle central. En présentant la gare Wilson de Prague, l'endroit d'où partit le Kindertransport qui séparait l'enfant Austerlitz de sa famille, le protagoniste raconte ses impressions d'adulte concernant ce lieu de la manière suivante: "Ce campement impossible à embrasser d'un seul regard était baigné d'une lumière proprement infernale, 
rouge lilas ${ }^{15}$.» Dans d'autres passages également, les lumières intérieures suscitent des associations avec les enfers. La présentation de Liverpool Street Station commence par une comparaison: la gare, dont le corps principal se trouve en dessous du niveau de la rue, est «l'un des lieux les plus sombres et les plus sinistres de Londres, une sorte de porte des Enfers $»^{16}$. En poursuivant le récit, on apprend que tout l'intérieur de la gare est envahi par la poussière et la crasse qui s'y accumulent depuis des centaines d'années. Le noir est par conséquent omniprésent, et les rayons de lumière s'y transforment en grisaille diffuse. Nous sommes loin de la pénombre d'une église gothique et des rayons de lumière qui contribuent à souligner l'ambiance sacrale de l'intérieur. Malgré les vitraux, les différentes sources lumineuses rendent menaçant l'espace de la gare. Au lieu de clarifier, d'ordonner, d'organiser le lieu, les lumières de la gare contribuent plutôt à désorienter le voyageur du roman, à rendre plus difficile toute prise de repères.

\section{Camouflage du réel}

Dès le milieu du XIX ${ }^{e}$ siècle, afin de compenser les bouleversements urbanistiques causés par les voies et les stations de trains, les architectes tendent à «camoufler la réalité fonctionnelle du nouvel instrument ferroviaire, à dissimuler à la vue du citadin la modernité industrielle du chemin de fer ${ }^{17}$. Ainsi, l'apparence des bâtiments de gares, de même que leurs décors ont pour rôle principal de faire oublier la destination de ces édifices. Dès lors, toute une série d'opérations entre en jeu afin de composer une image, donner une sensation et créer une atmosphère étrangères à l'idée de voyage et de déplacement mécanisé. Avec l'importance croissante des gares, les édifices deviennent de plus en plus complexes, répondant à des typologies diversifiées.

Selon les traités de l'époque, la gare « présente une composition dont le plan est la somme des espaces particuliers - le hall des arrivées et des départs ou "salle des pas perdus", la billetterie, le dépôt pour les bagages [...] - qui se succèdent selon les principes de composition enseignés à l'École des beaux-arts ${ }^{18}$. L'articulation des différents locaux devient donc une véritable problématique artistique dont le résultat répond parfois même moins à des objectifs fonctionnels qu'esthétiques. Vue en élévation, la gare "se réfère au répertoire stylistique néoclassique ou néogothique qui prévaut, et l'élément décoratif joue un rôle fondamental. Dans son ensemble, la gare adopte les dimensions et les définitions typologiques d'un véritable "palais urbain", caractérisé par la décoration particulière des façades et des espaces internes destinés au public. $»^{19}$ (ill. 5) Les palais marquent une continuité entre la ville et la gare, comme si le tissu habitable trouvait son expansion dans les locaux ferroviaires. 


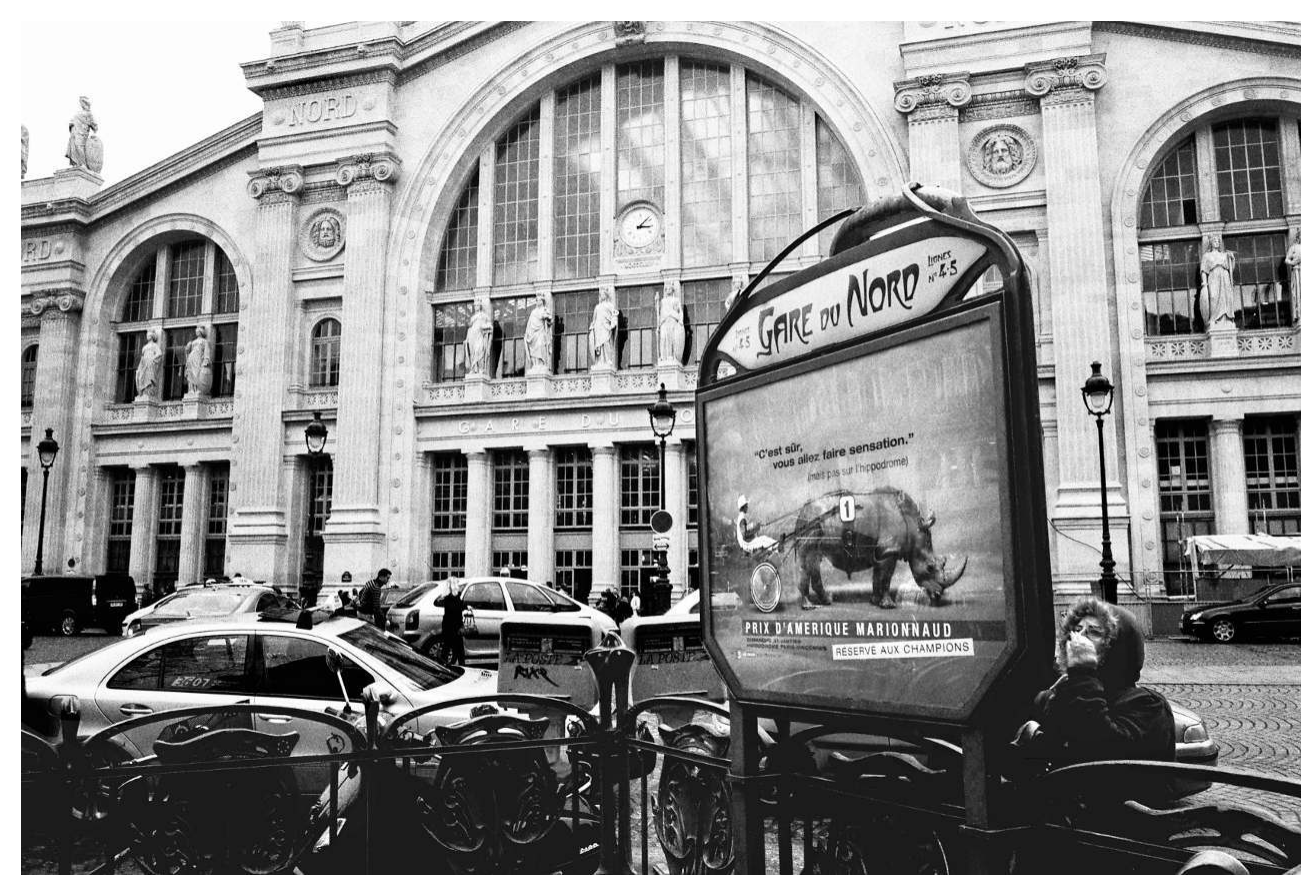

Cl. Imre Miszlay

36 À ce jeu de travestissement typologique s'ajoute une caractéristique très parlante, puisque la palette s'enrichit avec un vocabulaire théâtral. Afin de marquer l'entrée d'un axe de transport en ville en effet, les bâtiments des gares de la deuxième moitié du XIX ${ }^{\mathrm{e}}$ siècle devaient répondre, en plus des objectifs mentionnés ci-dessus, à un programme urbanistique spécifique. Ils étaient censés théâtraliser en quelque sorte l'arrivée des trains dans les centres-villes. L'aménagement des édifices ressemble ainsi à une mise en scène élaborée où les façades empruntent les caractéristiques d'un décor théâtral et fonctionnent comme une sorte de rideau de scène. L'organisation majestueuse de l'espace des salles des pas perdus transforme ces lieux en foyer d'opéra ${ }^{20}$. Cette orchestration du rituel du départ et de l'arrivée ainsi que la représentation des nouveaux pouvoirs industriels et politiques permettent de déceler un mécanisme fondamental des gares de cette période.

37 Le décor théâtral a donc pour objectif de souligner une scénographie du voyage, une mise en scène du pouvoir, un camouflage du bruit, de la pollution et de la rudesse des chemins de fer. Autrement dit, il s'agit de prétendre quelque chose, de donner une image ou de mythifier une situation, un acte. Le théâtre de la gare est donc le lieu de l'illusion, de la tromperie, du déguisement.

38 Qu'est-ce qu'un théâtre ? C'est un lieu qui permet la « mise en scène par des corps vivants des signes de pensées et de sentiments qui ne sont pas les leurs $»^{21}$. Or, à la gare, tout le monde est acteur et public à la fois, tout voyageur participe à cette mise en scène de l'illusion tout en étant trompé par elle. Jeu et vie, mirage et réalité ne font qu'un, les symboles ne sont plus représentés, mais habités (ill. 6). Par une entrée basée sur les principes de la représentation et de l'hypocrisie - car en fin de compte le lieu donne une apparence que le contenu ne suit pas - le sens et le déploiement de l'ensemble des espaces doivent être remis en question. La cathédrale ou le palais aristocratique ne sont que des éléments de décor de ce théâtre ; dès lors, les configurations faisant appel à des éléments 
d'un vocabulaire autre que celui des gares (telles que façades, nefs, rosaces) affichent des programmes architecturaux sans pouvoir en maintenir les cohérences. L'apparence de l'univers sacré, noble ou glorieux, sera discréditée par le reste.

III. 6 : Théâtre du départ

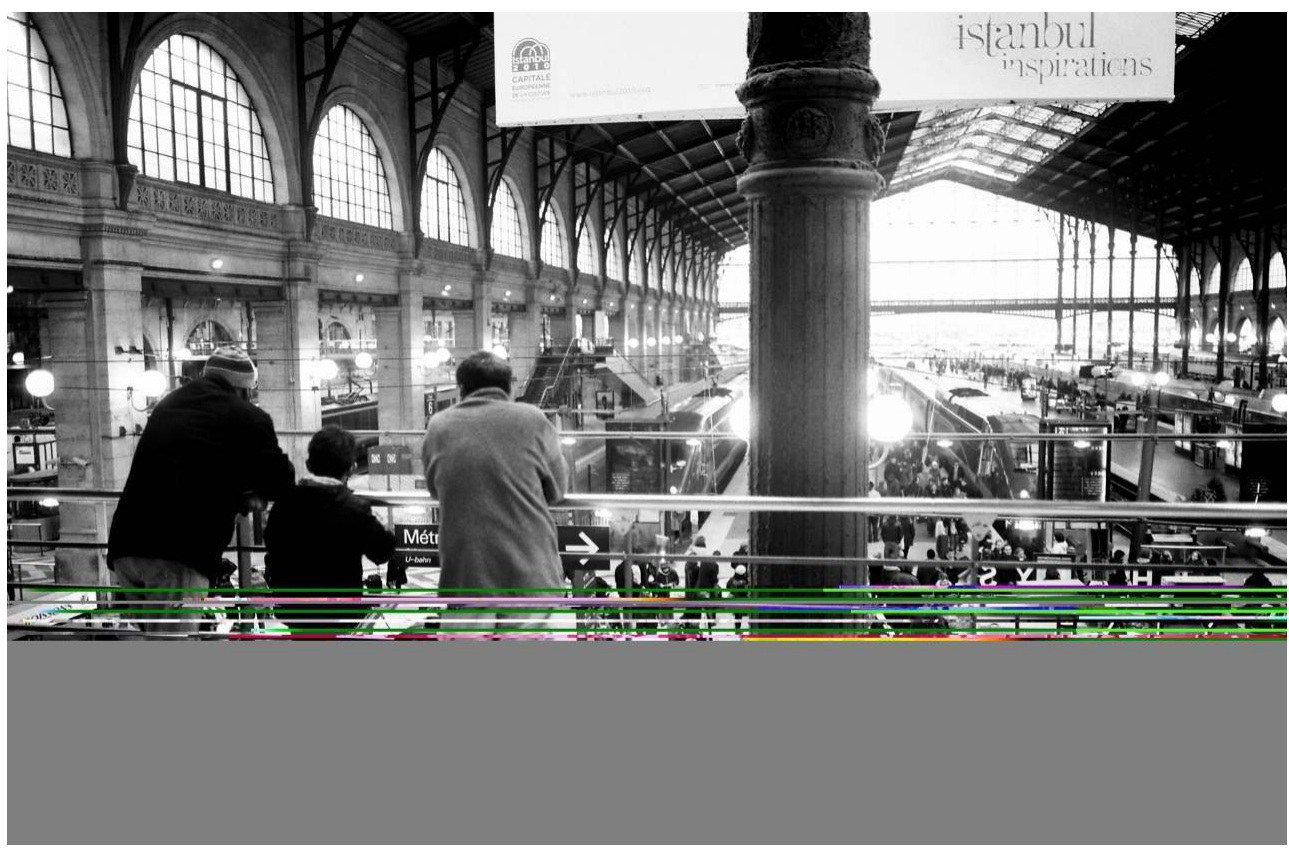

Cl. Imre Miszlay

\section{Le théâtre d'Austerlitz}

Curieusement, l'Austerlitz de Sebald suit la rhétorique théâtrale des gares. Autrement dit, l'intrigue se développe en fonction de tournants soulignant l'univers du déguisement, de la scène et des acteurs. À chaque fois que le personnage principal est sur le point d'apprendre quelque chose de son passé, les métaphores et les comparaisons théâtrales empêchent une identification claire de la vérité identitaire d'Austerlitz.

40 Il semble que le fait de situer l'histoire d'Austerlitz dans des gares entraîne des conséquences majeures sur l'ensemble du récit. C'est la logique des bâtiments ferroviaires, la scénographie des salles d'attente et des halls juxtaposés qui impose ses règles à l'histoire du roman. Les gares fonctionnent donc selon un procédé qui consiste à montrer une image qui n'est ensuite pas étayée par le contenu. Ainsi, le texte littéraire ne fait qu'obéir à l'architecture quand il décrit une série de variantes d'apparence trompeuse et de drames scéniques. La description d'éléments architecturaux ne sert donc pas d'illustration pour une intrigue prédominante, mais se soumet à une logique métatextuelle. La lecture sebaldienne des gares de la deuxième moitié du XIX ${ }^{\mathrm{e}}$ siècle se base donc sur une compréhension et une interprétation complexe de leur architecture, que l'histoire d'Austerlitz va refléter. Du rôle d'illustration, l'architecture des gares et sa structure rhétorique se dirigent vers celui de protagoniste auquel tout autre agent du récit devra se soumettre du point de vue du fonctionnement de ce dernier.

41 Un univers architectural ne se compose pas uniquement d'une succession ou juxtaposition de vues. Dans le cas de ces gares de la deuxième moitié du XIX ${ }^{e}$ siècle, la 
question suivante semble cruciale : «Si la connaissance des choses fait d'abord appel à leur vision, ce qui est évident pour l'architecture - cela suffirait-il pour le corps? $»^{22}$ Autrement dit, un espace basé sur l'illusion théâtrale est un univers où de multiples programmes doivent concorder pour maintenir le faux semblant. La crédibilité passe par une série d'expériences spatiales, faites par le corps, l'ouïe, l'odorat, la vue, etc.

Or, comme cela peut souvent se produire, l'expérience acoustique, visuelle et physique de l'espace ne s'étayent pas mutuellement ${ }^{23}$. On a vu que, du point de vue du programme esthétique, l'apparence d'une cathédrale ou d'un foyer théâtral n'est jamais confirmée par les autres agents de la perception ou de la compréhension qui contribuent à former l'expérience architecturale. C'est justement cette logique des gares du XIX ${ }^{e}$ siècle que les mécanismes du texte romanesque tentent d'imiter. Les conflits créateurs entre corps humains et corps de gare, les désorientations, l'égarement et la théâtralisation de l'illusion servent ainsi de base à une série de dialogues entre le narrateur et Austerlitz. L'un et l'autre tentent de comprendre l'histoire d'une vie, d'une époque et d'un héritage socio-culturel en se servant de ce guide: l'architecture des gares, comme cicérone de l'Histoire.

Austerlitz ne « lit » pas seulement l'architecture, il l'insère dans l'univers littéraire sans modifier les contraintes, les logiques de fonctionnement que la construction des gares implique : ceci ne tuera pas cela.

\section{NOTES}

1. Winfried Georg Sebald (1944-2001), une des figures majeures de la littérature contemporaine, consacre de nombreux romans et essais aux étrangers et aux émigrés; ses textes mêlent faits historiques, reportages, notes de voyages, fiction et photos en noir et blanc.

2. . Le personnage principal, Jacques Austerlitz est élevé dans un petit village du pays de Galles, par un pasteur calviniste. Adulte, il part à la recherche de son passé et de sa véritable identité. À travers des études architecturales de gares, il parvient à reconstituer au fur et à mesure son voyage d'enfance, lors duquel il a été sauvé par un Kindertransport qui l'avait éloigné de sa famille, de sa langue maternelle et de ses origines pragoises.

3. . Voir sur ce sujet l'analyse de Lewis Mumford, La Cité à travers l'histoire, Paris, Seuil, 1964, 781

p., p. 559-603.

4. . Winfried Georg Sebald, Austerlitz. Arles, Actes Sud, 2002, 349 p., p. 156-157.

5. . Bernard Tschumi, Architecture and Disjonction. The MIT Press, Cambridge/London, 1994, 268 p., p. 121-137.

6. . W. G. Sebald, Austerlitz. op. cit., p. 155.

7. Ibid., p. 159.

8. . Le Temps des gares [catalogue d'exposition], exposition conçue et réalisée par le Centre de création industrielle et le Centre national d'art et de culture Georges Pompidou. Paris, du 13 décembre 1978 au 9 avril 1979, Paris, Centre national d'art et de culture Georges Pompidou, Centre de création industrielle, 1978, 159 p., p. 9.

9. . Christian Sapin, « Rotunda », Monique Jannet, Christian Sapin (dir.), Guillaume de Volpiano et l'architecture des rotondes, Dijon, éd. universitaires de Dijon, 1996, 334 p., p. 15. 
10. . Voir l'analyse de B. Tschumi, Architecture and Disjonction. op. cit., p. 121-137.

11. . W. G. Sebald, op. cit., p. 14.

12. . John Zilcosky

ZILCOSKY John

, «Sebald

SEBALD Winfried Georg

's Uncanny Travels: The Impossibility of Getting Lost », J. J. Long

LONG J. J.

, Anne Whitehead

WHITEHEAD Anne

(eds), W. G. Sebald - A Critical Companion, Edinburgh, Edinburgh University Press, 2004, 239

p., p. 104

13. . John Zilcosky

ZILCOSKY John

, « Lost and Found: Disorientation, Nostalgia, and Holocaust Melodrama in Sebald

SEBALD Winfried Georg

's Austerlitz », Modern Language Notes, Volume 121, $\mathrm{n}^{\circ}$ 3, avril 2006, « German Issue », p. 688-689.

14. . W. G. Sebald, Austerlitz. op. cit., p. 115-116. C'est nous qui soulignons.

15. . Ibid., p. 259.

16. . Ibid., p. 154

17. . Le Temps des gares, op. cit., p. 41.

18. . Cristiana Mazzoni, Gares. Architectures 1990-2010. Arles, Actes Sud, 2001, 278 p., p. 24.

19. . Ibid., p. 24.

20. . Voir Le Temps des gares, op. cit., p. 33-34, VIII, IV. et Michel Ragon, L'Architecture des gares. Paris, éd. Denoël, 1984, 108 p., p. 23-24.

21. Jacques Rancière, Le Spectateur émancipé. Paris, éd. la Fabrique, 2008, 145 p., p. 60.

22. . Marc Perelman, Construction du corps, fabrique de l'architecture. Figures, histoire, spectacle. Une critique de l'ordre visuel moderne. [Saint-Maurice], éd. de la Passion, 1994, 222 p., p. 15.

23. Barry Blesser, Linda-Ruth Salter, Spaces speak, are you listening ? Experiencing aural architecture. Cambridge, MIT Press, 2007, 437 p., p. 3.

\section{RÉSUMÉS}

Parmi les textes littéraires contemporains, peu consacrent autant d'attention à l'architecture, à la description littéraire d'espaces architecturaux et à l'image photographique de constructions urbaines que le dernier roman de W. G. Sebald, intitulé Austerlitz. L'objectif du présent article consiste à examiner la représentation littéraire des espaces et des édifices évoqués dans le roman, et à voir comment le sens multiple de l'architecture influence l'organisation structurelle du texte. On examinera certains mécanismes de fonctionnement des gares et de leurs espaces intérieurs afin de voir comment le texte «lit» ces constructions et à quel point la logique architecturale parvient à déterminer celle de la narration et de la composition du roman. 
Among contemporary literary texts, few have consecrated as much attention to architecture, to the literary description of architectural spaces and to the photographic image of urban constructions as Austerlitz, W. G. Sebald's last novel. In this article, our purpose is to examine the literary description of spaces and buildings that appear in the novel; and, to see how the architecure's polysemy influences the structure of the novel. I will attempt to describe railway stations' (and their interior spaces) mechanisms of function, in order to understand how this text "reads" these constructions and to what point architectural logic is a determinating factor for the logic of the composition and of the narration.

In keinem zeitgenössischen literarischen Text wird Architektur so prächtig vor Augen gestellt wie in W.G.Sebalds letztem Roman „Austerlitz“, sei es durch die literarische Beschreibung von architektonischen Räumen wie auch durch fotografische Betrachtung der urbanen Bauten.

Dieser Aufsatz nimmt sich also vor, die literarische Darstellung der in Sebalds Roman erwähnten Räume und Bauten durchzustudieren. Daraus erweist sich, dass solch eine vielseitige Anschauung der Architektur die strukturelle Organisation des Textes beeinflusst hat. Die genaue Untersuchung von gewissen funktionellen Einrichtungen der Bahnhöfe und deren inneren Anlagen zeigt, dass der Text die Bauten sozusagen „liest“, so dass der Aufbau der Erzählung und die Verfassung des Romans der logischen Struktur der Architektur entsprechen.

\section{AUTEUR}

\section{JULIA NYIKOS}

Julia Nyikos, née en 1978, est docteur en esthétique, sciences et technologies des arts. Elle a été allocataire-monitrice, puis attachée temporaire d'enseignement et de recherche pour enseigner les théories de l'art à l'université Paris 8 , où elle a soutenu sa thèse de doctorat intitulée Paradigme commun dans la création architecturale et littéraire en 2009. Elle participe à des colloques internationaux en France et à l'étranger, et elle a organisé également des journées d'études, ainsi qu'un colloque international intitulé Photographie et corps politique à l'École des beaux-arts de Budapest en 2007. Elle a publié plusieurs articles, parmi lesquels « Le récit contemporain de Frank Gehry et de Claude Simon ", Archistorm, 2007, n 26 ; « L'expérience de l'histoire et de la photographie dans la littérature ", Magyar Lettre Internationale, été $2008, \mathrm{n}^{\circ} 69$. Elle poursuit ses recherches autour de la problématique de l'expérience de l'espace urbain en arts et en littérature. Adresse électronique : julianyikos@yahoo.fr 